\title{
In vivo confocal laser scanning microscopy imaging of skin inflammation: Clinical applications and research directions (Review)
}

\author{
MIHAELA ADRIANA ILIE ${ }^{1,2}$, CONSTANTIN CARUNTU ${ }^{3,4}$, DANIELA LIXANDRU ${ }^{2}$, MIRCEA TAMPA ${ }^{5}$, \\ SIMONA-ROXANA GEORGESCU ${ }^{5}$, MARIA-MAGDALENA CONSTANTIN ${ }^{6}$, CAROLINA CONSTANTIN ${ }^{7,8}$, \\ MONICA NEAGU $^{7-9}$, SABINA ANDRADA ZURAC ${ }^{8,10}$ and DANIEL BODA ${ }^{1,4}$
}

${ }^{1}$ Dermatology Research Laboratory, 'Carol Davila’ University of Medicine and Pharmacy; Departments of ${ }^{2}$ Biochemistry and ${ }^{3}$ Physiology, 'Carol Davila' University of Medicine and Pharmacy, 050474 Bucharest; ${ }^{4}$ Department of Dermatology, 'Prof. N. Paulescu' National Institute of Diabetes, Nutrition and Metabolic Diseases, 011233 Bucharest; ${ }^{5}$ Department of

Dermatology, 'Victor Babes' Hospital, 'Carol Davila' University of Medicine and Pharmacy, 030303 Bucharest;

${ }^{6}$ The 2nd Department of Dermatology, Colentina Clinical Hospital, 020125 Bucharest; ${ }^{7}$ Department of Immunology, 'Victor Babes' National Institute of Pathology, 050096 Bucharest; ${ }^{8}$ Department of Pathology, Colentina Clinical Hospital, 020125 Bucharest; ${ }^{9}$ Faculty of Biology, University of Bucharest, 050095 Bucharest; ${ }^{10}$ Department of Pathology, 'Carol Davila' University of Medicine and Pharmacy, 050474 Bucharest, Romania

Received August 20, 2018; Accepted October 5, 2018

DOI: $10.3892 / \mathrm{etm} .2018 .6981$

\begin{abstract}
In vivo confocal laser scanning microscopy (CLSM) is a novel imaging technique that provides noninvasive, morphological characterization of skin structures with a resolution that is very close to that of light microscopy. Moreover, as it allows repeated imaging of the same skin area at different time-points, it is an excellent method for monitoring disease course, response to treatment or specific stimuli and a path to study dynamic phenomena in real-time. To date, two different variants of in vivo CLSM have been authorized in dermatological field, namely the reflectance confocal microscopy predominantly for clinical diagnosis and the fluorescence confocal microscopy mainly for research purposes. This study describes the principles of in vivo CLSM technique, its role in the diagnosis and monitoring of inflammatory skin diseases, as well as some promising research directions to study the dynamics of skin inflammation using this method. In vivo CLSM evaluation of inflammatory dermatoses and of the skin inflammatory component in various diseases has an undoubted potential with broad applications ranging from clinical, morphological to experimental, functional studies involving the skin.
\end{abstract}

Correspondence to: Dr Constantin Caruntu, Department of Physiology, 'Carol Davila' University of Medicine and Pharmacy, 8 Eroii Sanitari Avenue, 050474 Bucharest, Romania

E-mail: costin.caruntu@gmail.com

Key words: in vivo, in real-time, reflectance confocal microscopy, skin inflammation

\section{Contents}

1. Introduction

2. In vivo confocal laser scanning microscopy imaging of inflammatory skin diseases

3. In vivo confocal laser scanning microscopy imaging for skin conditions associated with inflammation

4. In vivo confocal laser scanning microscopy imaging for investigation of skin inflammation components

5. Conclusions

\section{Introduction}

Skin inflammation has a key role in both physiological and pathological conditions. Its evaluation is of great potential to provide essential information regarding the association between inflammatory processes and systemic or cutaneous diseases. However, existing classical methods can themselves induce inflammation and are not suitable for studying the dynamic, in vivo components of skin inflammation (1).

Noninvasive, in vivo imaging tools have gained popularity in dermatology to overcome the burden and limitations of histopathological examination. In vivo confocal laser scanning microscopy (CLSM) is a novel imaging technique that provides the noninvasive, morphological and dynamic characterization of skin structures with a resolution that comes close to that of light microscopy, therefore performing a skin 'optical biopsy' (2). As it allows repeated imaging of the same skin area at different time-points, it is an excellent method for monitoring disease course, response to treatment or specific stimuli and a path to study dynamic phenomena in real-time (1-6). 
To date, two different variants of in vivo CLSM have been authorized in dermatological field, namely the reflectance confocal microscopy (RCM) predominantly for clinical diagnosis use and the fluorescence confocal microscopy mainly for studying skin penetration of various substances (7). In vivo RCM achieves contrast from backscattered light of various components of the skin, in their native state and it uses a laser with near-infrared wavelengths, enabling a maximum penetration depth of 200-300 $\mu \mathrm{m}$ that corresponds to the epidermis and upper dermis (8). The restricted depth of examination is the most recognized limitation of the currently commercially available confocal microscopes, but there are attempts to develop new devices that could overcome this drawback (9).

Skin components with high refractive index like melanin and keratin provide high contrast and strongly backscatter light. Therefore, cells containing melanin or keratin appear bright in RCM images (3). The usefulness of this novel technology has been recognized for the noninvasive investigation of melanocytic (10-12) and non-melanocytic lesions (13-16), of various inflammatory dermatologic conditions (17-25) and of various skin inflammatory processes (6). RCM can rapidly identify the pathological features of dermatoses with atypical clinical presentation with no associated pain or trauma for the patient (26).

As it allows repeated imaging of the same skin area at different time intervals, it has the advantage of monitoring disease progression, as well as treatment efficacy and side effects (27-29). In recent years, attention was turned to the study of dynamic processes such as wound healing $(30,31)$, skin aging (32), ultraviolet radiation (UVR)-induced alterations (33-35), in real-time assessment of blood flow in response to various topical stimuli $(6,36,37)$ or leucocyte migration $(1,4)$.

This study describes the role of in vivo RCM technique in the diagnosis and monitoring of inflammatory skin diseases, as well as some promising research directions to study the dynamics of skin inflammation using this method.

\section{In vivo confocal laser scanning microscopy imaging of inflammatory skin diseases}

Plaque psoriaris. Plaque psoriasis is a common, chronic inflammatory skin disorder (38), usually with a typical clinical presentation. Sometimes, to exclude other similar erythematous-squamous diseases and to confirm the clinical suspicion, a skin biopsy is needed, despite its invasiveness $(39,40)$. In early stages of the disease, the histopathological result may be equivocal and often cannot be differentiated from spongiotic dermatitis.

Characteristic micromorphological features of plaque psoriasis lesions can be easily identified with in vivo RCM, showing a high correlation with histology sections $(19,20,41)$. In the majority of cases, the stratum corneum is thickened (hyperkeratosis) and associates small dark nuclei within its bright cells (parakeratosis). Sometimes, clusters of highly refractile round to polygonal cells can be seen between the corneocytes and correspond to the diagnostic Munro's collections of neutrophils (42). Going deeper into the epidermis, a reduced or even absent granular layer (hypogranulosis) is observed, whereas stratum spinosum has an increased thickness (acanthosis). The horizontal RCM optical sections show an increase in the diameter $(>100 \mu \mathrm{m})$ and density of dermal papillae (papillomatosis), as well as dilated blood vessels surrounded by moderately refractile inflammatory cells in the superficial dermis (Fig. 1) (17-20). Moreover, in vivo RCM can be used for the objective assessment of the response to various treatments at microscopic level by performing serial determinations of the same skin area $(28,29,32)$.

Recently, our research group identified a method (43) for the objective assessment of psoriasis vulgaris lesions using in vivo RCM technique that is potentially applicable to clinical studies and monitoring the evolution of lesions under treatment.

Lichen planus. Lichen planus is an inflammatory mucocutaneous disease with still unclear etiopathogenesis. In vivo RCM has been used for the noninvasive evaluation of cutaneous lichen planus and enabled the identification of its distinctive features, with a good correlation with histological findings (Fig. 2) (21). Confocal features of the epidermal layers include increased intercellular spaces (spongiosis), large, polygonal cells (hypergranulosis) disposed in a thickened wedge-shaped granular cell layer (corresponding to Wickham's striae) and the presence of grouped round-to-polygonal bright cells (inflammatory infiltrates).

Due to the extensive inflammatory cell infiltrate at the epidermal-dermal junction level, the replacement of the bright ring-like structures around dermal papillae with smeared refractile rings can be observed (vacuolar degeneration of the basal layer). Large bright, plump, oval to stellate cells (melanophages) and dilated blood vessels can be seen in the superficial dermis $(21,44)$. In vivo RCM represents a useful tool for the noninvasive diagnosis of lichen planus, but further studies are necessary in order to distinguish between different subtypes of interface dermatitis (21).

Discoid lupus erythematosus (DLE). DLE is an inflammatory condition that can be difficult to distinguish clinically from other erythematosquamous skin diseases. In vivo RCM evaluation of DLE lesions enables the identification of key diagnostic features seen in histopathological samples including epidermal atrophy, interface changes, as well as epidermal, dermal and periadnexial inflammatory cell infiltration (Fig. 3) (22). The maximum depth of imaging limited to the upper dermis and the inability to distinguish lymphocytes from other inflammatory cells are disadvantages of RCM examination in DLE. However, combining dermoscopy with RCM has an important role in choosing the apropriate biopsy site for more histologic diagnostic criteria $(22,45)$.

In vivo RCM proved to be useful also in the diagnosis of inflammatory dermatoses with special localizations like the scalp, including alopecia areata $(23,24)$, lichen planopilaris and DLE secondary scarring alopecia (45). RCM criteria for these inflammatory skin conditions strongly correlate with histologic features and are sensitive enough to differentiate between entities with similar clinical presentation (25). Also, during follow-up of lesions, some micromorphological features helped in choosing the appropriate therapeutic option.

\section{In vivo confocal laser scanning microscopy imaging for skin conditions associated with inflammation}

Cutaneous wound healing. Cutaneous wound healing is a continuously expanding research area and various noninvasive 

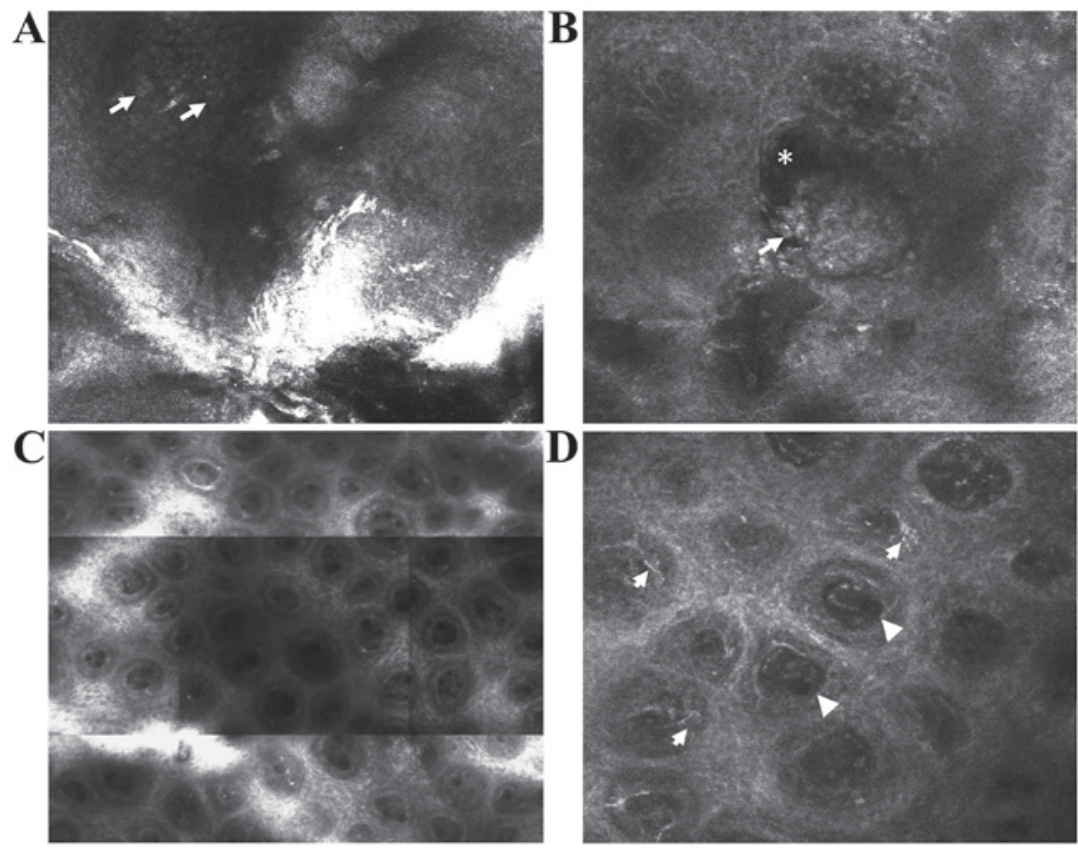

Figure 1. In vivo RCM features of psoriasis. (A) RCM image $(0.5 \times 0.5 \mathrm{~mm})$ showing round to polygonal, nucleated bright cells $(\rightarrow)$ in the cornified layer corresponding to parakeratotic keratinocytes. (B) RCM image $(0.5 \times 0.5 \mathrm{~mm})$ showing clustered refractile roundish structures $(\rightarrow)$ associated with dark areas $(*)$ corresponding to accumulation of leukocytes in the cornified layer (Munro micro abscesses) or in the upper portion of the spinous layer (spongiform pustules of Kogoj). (C) RCM mosaic (1x1 mm) showing increased density of dermal papillae. (D) RCM image (0.5x0.5 mm) showing enlarged dermal papillae lacking bright ring of basal cells occupied by dilated blood vessels $(\bullet)$ and surrounded by moderately refractile dendritic inflammatory cells $(\rightarrow)$.
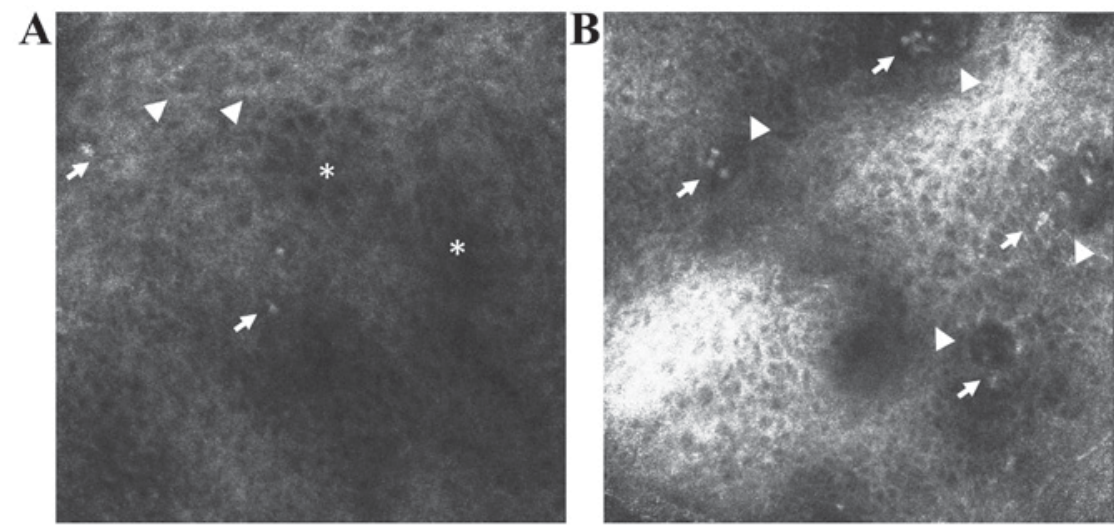

Figure 2. In vivo RCM features of lichen planus. (A) RCM image $(0.5 \mathrm{x} 0.5 \mathrm{~mm})$ at the level of the granular-spinous layer showing increased intercellular spaces (spongiosis) (*), large, polygonal cells (hypergranulosis in a wedge-shaped pattern that corresponds to the Wickham's striae) ( $\bullet$ ) and inflammatory cells that appear as roundish bright structures $(\rightarrow)$. (B) RCM image $(0.5 \times 0.5 \mathrm{~mm})$ at the level of the epidermal-dermal junction showing non-edged and non-rimmed dermal papillae $(\bullet)$ due to inflammatory cell infiltrate $(\rightarrow)$.

imaging techniques have been evaluated for their applicability in monitoring healing of skin wounds (46). Among these techniques, in vivo RCM was able to visualize the inflammatory, vascular and tissue remodeling features associated with cutaneous wound repair in real-time, at different time intervals (30). A recent study performed by our research group on BALB/c mice showed that in vivo RCM is able to assess the extent of wound dehiscence, restoration of the normal honeycombed pattern of epidermis and of dermal fibro-elastic structures, as well as the aspect of dermal blood vessels and the presence of inflammatory cells (unpublished data) (Fig. 4).

Moreover, it can provide an objective and noninvasive assessment of burn wounds gravity either alone, with the advantage of its near cellular resolution (47), or combined with optical coherence tomography for a better depth penetration (48). Moreover, in vivo RCM can predict the healing course of burn wounds of indeterminate depth based on serial determinations of microcirculation, morphology and inflammatory cell traffic (49). This noninvasive high-resolution technique was also able to assess the effects of changes in cutaneous microcirculation on tissue morphology during burn wound healing (31).

Skin aging. Skin aging is subject of increased research in dermatology and cosmetology reflected in the continuously development of products and methods that could prevent or reverse this complex inevitable process. In vivo CLSM proved to be a reliable method for assessment of skin aging and revealed 

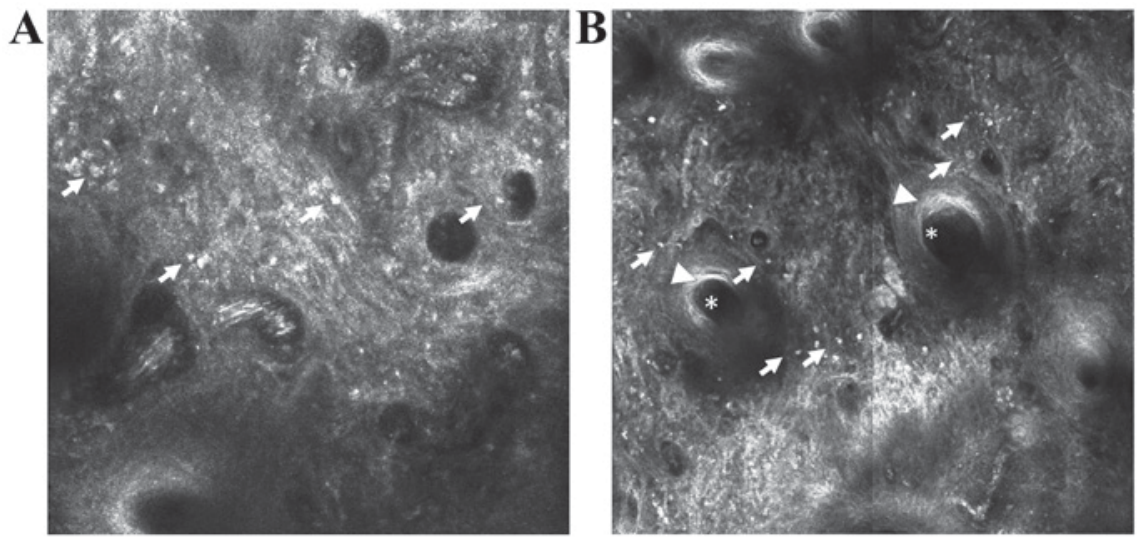

Figure 3. In vivo RCM features of discoid lupus erythematosus. (A) RCM mosaic (1x1 mm) at the epidermal-dermal junction level showing disappearance of the papillary rings due to infiltrates of inflammatory cells $(\rightarrow)$. (B) RCM mosaic $(1 \times 1 \mathrm{~mm})$ showing dillated follicle $(*)$ with infundibular hyperkeratosis $(\bullet)$ surrounded by inflammatory cells $(\rightarrow)$.
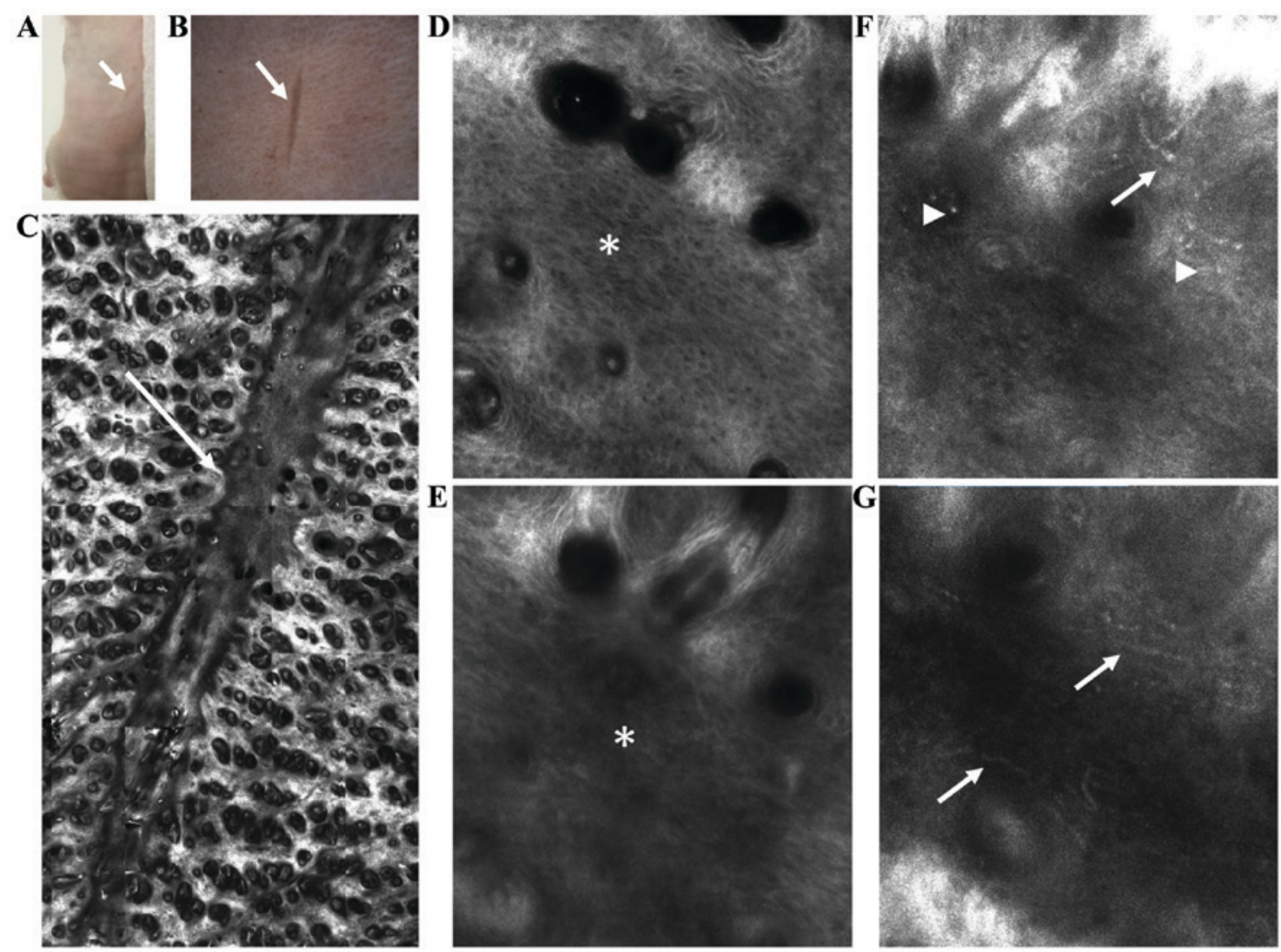

Figure 4. Evaluation of skin wound healing in BALB/c mice by in vivo RCM. (A) Clinical aspect of the wound ( $\rightarrow$ ). (B) Corresponding dermoscopic image of lesional skin $(\rightarrow)$. (C) RCM of skin lesion showing a clear demarcation line between the scar and perilesional areas $(\rightarrow)$. (D) Lesional epidermis with polymorphic aspect (*) different as compared to the normal honeycombed pattern of the perilesional areas. (E) Superficial dermis with thin fibers creating a blurry aspect in the lesional area $(*)$. $(\mathrm{F}$ and $\mathrm{G})$ Tortuous blood vessels with increased blood flow $(\rightarrow)$ and bright inflammatory cells $(\bullet)$ in perilesional dermis.

the presence of keratinocyte alterations, irregular pigmentation and increased compactness of collagen fibers that became more pronounced with aging (32). This noninvasive method offers potential applications in choosing the appropriate antiaging method and testing cosmetic treatment efficacy.

UVR-induced alterations. UVR-induced alterations are responsible for extrinsic aging and play an important role in skin cancer development (33). In vivo RCM was able to identify and monitor UVR produced skin alterations beginning with skin inflammation, followed by the appearance of microvesicles, apoptotic keratinocytes and activated melanocytes and finally, loss of the epidermal structure (34). Specifically, ultraviolet A (UVA) radiation effects upon the skin were analyzed using this noninvasive technique, confirming that UVA-induced accelerated blood flow is a prerequisite for the development of immediate and delayed tanning (50). Another study showed the negative effects of sun 

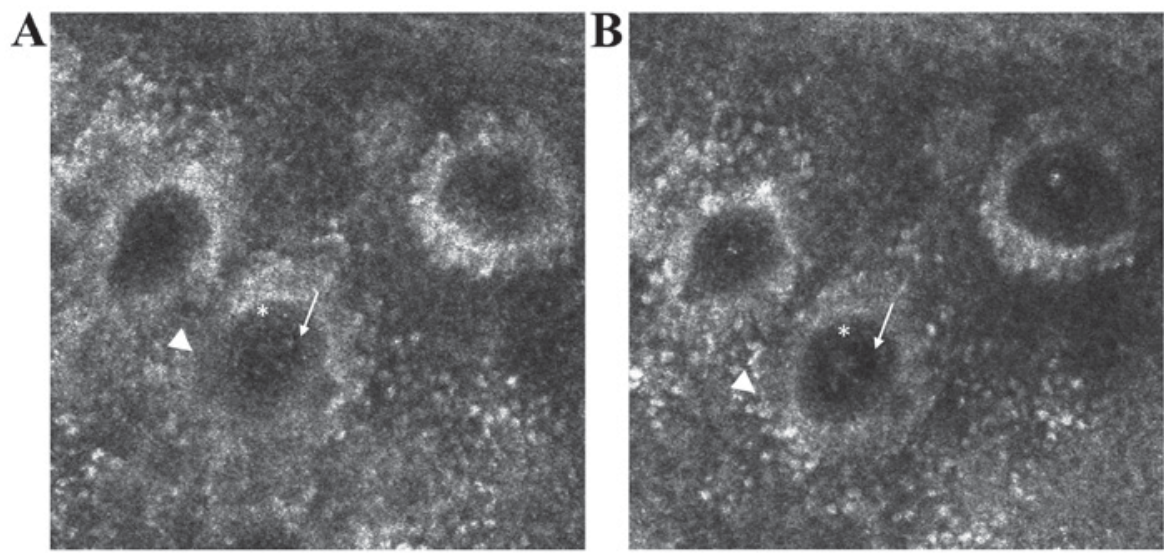

Figure 5. In vivo RCM sequential images $(0.25 \times 0.25 \mathrm{~mm})$ at the level of the epidermal-dermal junction, 0 min (A) and 25 min (B) after the administration of topical capsaicin. Dermal capillaries appear as black holes $(\rightarrow)$ inside dermal papillae, dark roundish areas $(*)$ that are surrounded by bright circles $(\bullet)$, corresponding to the epidermal basal layer. In real-time blood cells can be observed as moving bright elements inside the lumina of dermal capillaries. Dilation of dermal capillaries and flow of blood cells inside their lumina can be easily observed after 25 min of topical capsaicin.

exposure upon epidermal architecture, dermal collagen and overall skin thickness (35).

Moreover, a recent study demonstrated the utility of RCM for an objective quantitative definition of sensitive skin and showed that disarranged as well as reduced honeycomb pattern depth and the presence of spongiform edema were predominant confocal features for this type of skin (51).

\section{In vivo confocal laser scanning microscopy imaging for investigation of skin inflammation components}

Leukocyte migration. Leukocyte migration or relocation is a synchronized active process through which both innate and adaptive immune system work to resolve a distally located inflammation or injury (52). Although intensively studied as important immune defenders, the complex voyage of leukocytes, especially neutrophils, alongside the blood vessel claims new imaging methods such as CLSM, or even a three-dimensional (3D) approach, in order to reveal their in-depth spatiotemporal scene in vessels and surrounding tissues. Thus, acknowledged to confocal fluorescent microscopy it becomes possible to envisage intravascular migration of neutrophils, extravasation as well as interstitial passage thorough peripheral vessels as a prompt response to physically damaged tissue, bacteria and virus invaders in order to reduce the injury and preserve the body homeostasis (53).

Why is a 3D imaging approach so worthy in monitoring a fine cellular process like leukocyte transmigration cascade? Firstly, it catches cellular aspects which, although important, cannot be detected with other in vitro investigation methods. For instance, besides endothelial cells pericytes are also involved which are rarely included and examined by in vitro methods, although they express key adhesion molecules (e.g., VCAM-1), are an integrant part of the vessel wall and perform an active role in transmigration (54). Secondly, a 3D approach would clasp in real-time the timing process; for example, in vitro detection of neutrophils passage was reported within 2 min while in vivo methods reported 15-45 min for neutrophil migration through endothelial cells (55).
These aspects become very important when, for instance the effect of a stimulus or a therapeutic agent on leukocyte functions is tested, and also when investigator would directly track the whole picture of leukocyte behavior in a physiologically milieu including all junctions and intercellular interactions. The final step of leukocyte/neutrophil migration (extravasation) was evidenced and reported quite recently; thus, the uropod elongation as final step of extravasation was imaged by multiphoton intravital microscopy in cremaster muscle leukocytes of CD18-mCFP KI mice following neutrophil stimulation (CXCL2, fMLP and TNF) and Texas Red-dextran staining of blood vessels. Images reveal that during extravasation the $\mathrm{CD}_{18}{ }^{+}$neutrophils marked with Alexa Fluor 488-anti-Gr1 antibody migrated from cremaster venules are about 4 times elongated compared with rolling and crawling leukocytes (56). Thus, migration process imaging provides valuable data regarding accurate function and regulation of leukocyte recruitment to injury site with potential impact in therapeutic purposes (4).

Capillary blood flow. Capillary blood flow within dermal vascularization is a parameter that can be easily monitored in real-time, in vivo with RCM imaging due to brightly reflecting of blood cells. Several factors are usually recorded, thus, the quantitative blood cell flow per minute is measured, taking into account the number of capillary loop and multiple fields of view digitally measured for a fixed time-point (e.g., every $30 \mathrm{sec}$ ). Further, the capillary loop diameter and the density of dermal capillaries per area are registered in the dermal papillae of the epidermal-dermal junction, where multiple fields of view are captured in real-time images (36). In addition, in real-time investigation of skin blood flow changes induced by topical capsaicin using RCM was proposed as a research model to test neurovascular reactivity (5).

An alternative to RCM is two-photon excitation microscopy that provides advantages for $3 \mathrm{D}$ and deep tissue imaging. A widespread application of this tool is to evaluate the blood flow via blood cell velocity and brightness, these measurements being very useful in setting different experimental models such as stroke, embryo development (57) or different 
skin cancers (basal cell carcinoma, squamous cell carcinoma) where morphological and functional assessments of skin layers are primarily made by combining techniques as laser Doppler flowmetry and RCM (58).

Skin reactivity to topical stimuli. Skin reactivity to certain local stimuli could be an excellent model for revising various skin conditions through evaluation of the inflammatory process at the skin level $(6,37)$. Neurogenic inflammation was experimentally locally induced by capsaicin and further CLSM in reflectance mode enabled the assessment of the cutaneous micro-vascularization (Fig. 5). This could represent an important research model for studying the link between cutaneous diseases and the nervous system (6).

As an innovative non-invasive method for in vivo imaging of skin structure, CLSM could be successfully applied in dermatological fields less studied until now in terms of imaging, such as experimental contact dermatitis. Such attempts have been made for almost one decade when in situ imaging of skin reactions produced by sodium lauryl sulphate and pelargonic acid as experimental irritants was reported; the group describes extended cell boundaries, keratinocyte swelling (pelargonic acid) and induction of parakeratosis within the stratum corneum (sodium lauryl sulphate) (59).

CLSM was applied also in a recent investigation in helping the patch test interpretations, a test known as the gold standard for contact dermatitis validation, and thus to differentiate very precisely between allergic, irritant, and equivocal patch test reactions; in this study, in vivo CLSM assessment revealed that $40 \%$ from equivocal reactions displayed confocal patterns in line with the positive allergic reactions patterns (60).

\section{Conclusions}

In vivo CLSM is a novel imaging technique that provides the morphological and dynamic characterization of skin structures with a high, quasi-microscopic resolution. The non-invasive character of the examination and the possibility to evaluate the same skin area at different time-points make in vivo CLSM a useful tool in the diagnosis and monitoring of inflammatory skin diseases. Moreover, it is an excellent method to study in real-time the dynamic components of skin inflammation, response to treatment or specific stimuli with broad applications ranging from clinical to experimental, functional studies involving the skin. Studying the components of skin inflammation is also of great potential to unravel pathways in the pathogenesis of diseases associated with skin inflammation and might contribute to the development of new treatment strategies.

\section{Acknowledgements}

Not applicable.

\section{Funding}

This study was partially supported by a grant of the Romanian Ministry of Research and Innovation, CCCDI-UEFISCDI (project nos. 61PCCDI/2018 PN-III-P1-1.2-PCCDI-2017-034 and PN-III-P2-2.1-BG-2016-0443), within PNCDI-III.

\section{Availability of data and materials}

Not applicable.

\section{Authors' contributions}

MAI, CCa, DL, MT, SRG, MMC, CCo, MN, SAZ and DB contributed equally to acquisition, analysis and systematization of data, manuscript writing and critical revision of it for important intellectual content. All authors read and approved the final version of the manuscript.

\section{Ethics approval and consent to participate}

Not applicable.

\section{Patient consent for publication}

Not applicable.

\section{Competing interests}

The authors declare that they have no competing interests.

\section{References}

1. Peppelman M, Wolberink EA, Gerritsen MJ, van de Kerkhof PC and van Erp PE: Application of leukotriene B4 and reflectance confocal microscopy as a noninvasive in vivo model to study the dynamics of skin inflammation. Skin Res Technol 21: 232-240, 2015.

2. Diaconeasa A, Boda D, Neagu M, Constantin C, Căruntu C, Vlădău L and Guţu D: The role of confocal microscopy in the dermato-oncology practice. J Med Life 4: 63-74, 2011.

3. Rajadhyaksha M, Grossman M, Esterowitz D, Webb RH and Anderson RR: In vivo confocal scanning laser microscopy of human skin: Melanin provides strong contrast. J Invest Dermatol 104: 946-952, 1995.

4. González S, Sackstein R, Anderson RR and Rajadhyaksha M: Real-time evidence of in vivo leukocyte trafficking in human skin by reflectance confocal microscopy. J Invest Dermatol 117: 384-386, 2001

5. Ghiță MA, Căruntu C, Rosca AE, Căruntu A, Moraru L, Constantin C, Neagu M and Boda D: Real-time investigation of skin blood flow changes induced by topical capsaicin. Acta Dermatovenerol Croat 25: 223-227, 2017.

6. Căruntu C and Boda D: Evaluation through in vivo reflectance confocal microscopy of the cutaneous neurogenic inflammatory reaction induced by capsaicin in human subjects. J Biomed Opt 17: 085003, 2012.

7. Meyer LE, Otberg N, Sterry W and Lademann J: In vivo confocal scanning laser microscopy: Comparison of the reflectance and fluorescence mode by imaging human skin. J Biomed Opt 11: 044012, 2006.

8. Skvara H, Plut U, Schmid JA and Jonak C: Combining in vivo reflectance with fluorescence confocal microscopy provides additive information on skin morphology. Dermatol Pract Concept 2: 3-12, 2012

9. Izatt JA, Kulkarni MD, Hsing-Wen W, Kobayashi K and Sivak MV: Optical coherence tomography and microscopy in gastrointestinal tissues. IEEE J Sel Top Quantum Electron 2: 1017-1028, 1996.

10. Pellacani G, Guitera P, Longo C, Avramidis M, Seidenari S and Menzies S: The impact of in vivo reflectance confocal microscopy for the diagnostic accuracy of melanoma and equivocal melanocytic lesions. J Invest Dermatol 127: 2759-2765, 2007.

11. Guida S,Longo C, Casari A, Ciardo S, Manfredini M, Reggiani C, Pellacani $G$ and Farnetani F: Update on the use of confocal microscopy in melanoma and non-melanoma skin cancer. G Ital Dermatol Venereol 150: 547-563, 2015 (In Italian).

12. Guida S, Longo C, Casari A, Ciardo S, Manfredini M, Reggiani C, Pellacani G and Farnetani F: Distinct melanoma types based on reflectance confocal microscopy. Exp Dermatol 23: 414-418, 2014. 
13. Ghita MA, Caruntu C, Rosca AE, Kaleshi H, Caruntu A, Moraru L, Docea AO, Zurac S, Boda D, Neagu M, et al: Reflectance confocal microscopy and dermoscopy for in vivo, non-invasive skin imaging of superficial basal cell carcinoma. Oncol Lett 11: 3019-3024, 2016.

14. Căruntu C, Boda D, Guţu DE and Căruntu A: In vivo reflectance confocal microscopy of basal cell carcinoma with cystic degeneration. Rom J Morphol Embryol 55: 1437-1441, 2014.

15. Lupu M, Caruntu C, Solomon I, Popa A, Lisievici C, Draghici C, Papagheorghe L, Voiculescu V and Giurcaneanu C: The use of in vivo reflectance confocal microscopy and dermoscopy in the preoperative determination of basal cell carcinoma histopathological subtypes. Dermatovenerologia 62: 265-275, 2017.

16. Lupu M, Caruntu A, Caruntu C, Boda D, Moraru L, Voiculescu V and Bastian A: Non-invasive imaging of actinic cheilitis and squamous cell carcinoma of the lip. Mol Clin Oncol 8: 640-646, 2018.

17. Białek-Galas K, Wielowieyska-Szybińska D, Dyduch G and Wojas-Pelc A: The use of reflectance confocal microscopy in selected inflammatory skin diseases. Pol J Pathol 66: 103-108, 2015

18. Wolberink EA, van Erp PE, Teussink MM, van de Kerkhof PC and Gerritsen MJ: Cellular features of psoriatic skin: Imaging and quantification using in vivo reflectance confocal microscopy. Cytometry B Clin Cytom 80: 141-149, 2011.

19. Ardigo M, Cota C, Berardesca E and González S: Concordance between in vivo reflectance confocal microscopy and histology in the evaluation of plaque psoriasis. J Eur Acad Dermatol Venereol 23: 660-667, 2009.

20. González S, Rajadhyaksha M, Rubinstein G and Anderson RR Characterization of psoriasis in vivo by reflectance confocal microscopy. J Med 30: 337-356, 1999.

21. Moscarella E, González S, Agozzino M, Sánchez-Mateos JL, Panetta C, Contaldo M and Ardigò M: Pilot study on reflectance confocal microscopy imaging of lichen planus: A real-time, non-invasive aid for clinical diagnosis. J Eur Acad Dermato Venereol 26: 1258-1265, 2012

22. Ardigò M, Maliszewski I, Cota C, Scope A, Sacerdoti G, Gonzalez S and Berardesca E: Preliminary evaluation of in vivo reflectance confocal microscopy features of Discoid lupus erythematosus. Br J Dermatol 156: 1196-1203, 2007.

23. Ardigò M, Tosti A, Cameli N, Vincenzi C, Misciali C and Berardesca E: Reflectance confocal microscopy of the yellow dot pattern in alopecia areata. Arch Dermatol 147: 61-64, 2011.

24. Rudnicka L, Olszewska M and Rakowska A: In vivo reflectance confocal microscopy: Usefulness for diagnosing hair diseases J Dermatol Case Rep 2: 55-59, 2008.

25. Koller S, Gerger A, Ahlgrimm-Siess V, Weger W, Smolle J and Hofmann-Wellenhof R: In vivo reflectance confocal microscopy of erythematosquamous skin diseases. Exp Dermatol 18: 536-540, 2009.

26. Ma J, Zhang X, Lv Y, Zhao C, Li Q, Yang X and Zhao J: Clinical application of confocal laser scanning microscopy for atypical dermatoses. Cell Biochem Biophys 73: 199-204, 2015.

27. González S, Sánchez V, González-Rodríguez A, Parrado C and Ullrich M: Confocal microscopy patterns in nonmelanoma skin cancer and clinical applications. Actas Dermosifiliogr 105 446-458, 2014

28. Wolberink EA, van Erp PE, de Boer-van Huizen RT, van de Kerkhof PC and Gerritsen MJ: Reflectance confocal microscopy: An effective tool for monitoring ultraviolet B phototherapy in psoriasis. Br J Dermatol 167: 396-403, 2012.

29. Ardigò M, Agozzino M, Longo C, Conti A, Di Lernia V, Berardesca E and Pellacani G: Psoriasis plaque test with confocal microscopy: Evaluation of different microscopic response pathways in NSAID and steroid treated lesions. Skin Res Technol 19: 417-423, 2013.

30. Lange-Asschenfeldt S, Bob A, Terhorst D, Ulrich M, Fluhr J, Mendez G, Roewert-Huber HJ, Stockfleth E and Lange-Asschenfeldt B: Applicability of confocal laser scanning microscopy for evaluation and monitoring of cutaneous wound healing. J Biomed Opt 17: 076016, 2012.

31. Altintas AA, Altintas MA, Ipaktchi K, Guggenheim M, Theodorou P, Amini P and Spilker G: Assessment of microcirculatory influence on cellular morphology in human burn wound healing using reflectance-mode-confocal microscopy. Wound Repair Regen 17: 498-504, 2009.

32. Longo C, Casari A, Beretti F, Cesinaro AM and Pellacani G: Skin aging: In vivo microscopic assessment of epidermal and dermal changes by means of confocal microscopy. J Am Acad Dermatol 68: e73-e82, 2013.
33. Solovastru LG, Vâta D, Statescu L, Constantin MM and Andrese E: Skin cancer between myth and reality, yet ethically constrained. Rev Rom Bioet 12: 47-52, 2014.

34. Koller S, Inzinger M, Rothmund M, Ahlgrimm-Siess V, Massone C, Arzberger E, Wolf P and Hofmann-Wellenhof R: UV-induced alterations of the skin evaluated over time by reflectance confocal microscopy. J Eur Acad Dermatol Venereol 28: 1061-1068, 2014.

35. Haytoglu NS, Gurel MS, Erdemir A, Falay T, Dolgun A and Haytoglu TG: Assessment of skin photoaging with reflectance confocal microscopy. Skin Res Technol 20: 363-372, 2014.

36. Altintas MA, Altintas AA, Guggenheim M, Steiert AE, Aust MC, Niederbichler AD, Herold $C$ and Vogt PM: Insight in human skin microcirculation using in vivo reflectance-mode confocal laser scanning microscopy. J Digit Imaging 23: 475-481, 2010.

37. Altintas AA, Guggenheim M, Oezcelik A, Gehl B, Aust MC and Altintas MA: Local burn versus local cold induced acute effects on in vivo microcirculation and histomorphology of the human skin. Microsc Res Tech 74: 963-969, 2011.

38. Caruntu C, Boda D, Dumitrascu G, Constantin C and Neagu M: Proteomics focusing on immune markers in psoriatic arthritis. Biomarkers Med 9: 513-528, 2015.

39. Schön MP and Boehncke WH: Psoriasis. N Engl J Med 352: 1899-1912, 2005

40. Wu H, Shapiro B and Harrist TJ: Noninfectious erythematous, popular, and squamous diseases. Psoriasis. In: Lever's Histopathology of the Skin. 9th edition. Elder DE, Elenitzas R, Johnson BL and Murphy GF (eds). Lippincott Williams \& Wilkins, Philadelphia, PA, pp59-60, 2005.

41. Căruntu C, Boda D, Căruntu A, Rotaru M, Baderca F and Zurac S: In vivo imaging techniques for psoriatic lesions. Rom J Morphol Embryol 55: 1191-1196, 2014.

42. Zhong LS, Wei ZP and Liu YQ: Sensitivity and specificity of Munro microabscess detected by reflectance confocal microscopy in the diagnosis of psoriasis vulgaris. J Dermatol 39: 282-283, 2012

43. Batani A, Brănișteanu DE, Ilie MA, Boda D, Ianosi S, Ianosi G and Caruntu C: Assessment of dermal papillary and microvascular parameters in psoriasis vulgaris using in vivo reflectance confocal microscopy. Exp Ther Med 15: 1241-1246, 2018.

44. González S: Lichen planus. In: Reflectance Confocal Microscopy in Dermatology: Fundamentals and Clinical Applications. Grupo Aula Medica, Madrid, pp25-26, 2012.

45. Agozzino M, Tosti A, Barbieri L, Moscarella E, Cota C, Berardesca $\mathrm{E}$ and Ardigò M: Confocal microscopic features of scarring alopecia: Preliminary report. Br J Dermatol 165: 534-540, 2011.

46. Mani R: Science of measurements in wound healing. Wound Repair Regen 7: 330-334, 1999.

47. Altintas MA, Altintas AA, Knobloch K, Guggenheim M, Zweifel CJ and Vogt PM: Differentiation of superficial-partial vs. deep-partial thickness burn injuries in vivo by confocal-laser-scanning microscopy. Burns 35: 80-86, 2009.

48. Iftimia N, Ferguson RD, Mujat M, Patel AH, Zhang EZ, Fox W and Rajadhyaksha M: Combined reflectance confocal microscopy/optical coherence tomography imaging for skin burn assessment. Biomed Opt Express 4: 680-695, 2013.

49. Altintas AA, Guggenheim M, Altintas MA, Amini P, Stasch T and Spilker G: To heal or not to heal: Predictive value of in vivo reflectance-mode confocal microscopy in assessing healing course of human burn wounds. J Burn Care Res 30: 1007-1012, 2009.

50. Yamashita T, Akita H, Astner S, Miyakawa M, Lerner EA and González S: In vivo assessment of pigmentary and vascular compartments changes in UVA exposed skin by reflectance-mode confocal microscopy. Exp Dermatol 16: 905-911, 2007.

51. Ma YF, Yuan C, Jiang WC, Wang XL and Humbert P: Reflectance confocal microscopy for the evaluation of sensitive skin. Skin Res Technol 23: 227-234, 2017.

52. Yadav R, Larbi KY, Young RE and Nourshargh S: Migration of leukocytes through the vessel wall and beyond. Thromb Haemost 90: 598-606, 2003.

53. Park SA and Hyun YM: Neutrophil extravasation cascade: What can we learn from two-photon intravital imaging? Immune Netw 16: 317-321, 2016.

54. Proebstl D, Voisin M-B, Woodfin A, Whiteford J, D'Acquisto F, Jones GE, Rowe D and Nourshargh S: Pericytes support neutrophil subendothelial cell crawling and breaching of venular walls in vivo. J Exp Med 209: 1219-1234, 2012. 
55. Stein B, Khew-Goodall Y, Gamble J and Vadas MA: Transmigration of leukocytes. In: Endothelium in Clinical Practice: Source and Target of Novel Therapies. Rubanyi GM and Dzau VJ (eds). Marcel Dekker, Inc., New York, NY, pp149-202, 1997.

56. Hyun YM, Sumagin R, Sarangi PP, Lomakina E, Overstreet MG, Baker CM, Fowell DJ, Waugh RE, Sarelius IH and Kim M: Uropod elongation is a common final step in leukocyte extravasation through inflamed vessels. J Exp Med 209: 1349-1362, 2012.

57. Benninger RK and Piston DW: Two-photon excitation microscopy for the study of living cells and tissues. Curr Protoc Cell Biol Chapter 4: 1-24, 2013. doi: 10.1002/0471143030. cb0411s59.

58. Mowla A, Taimre T, Lim YL, Bertling K, Wilson SJ, Prow TW, Soyer $\mathrm{H}$ and Rakić A: Concurrent reflectance confocal microscopy and laser doppler flowmetry to improve skin cancer imaging: A Monte Carlo model and experimental validation. Sensors (Basel) 16: 1411, 2016.
59. Suihko C and Serup J: Fluorescence confocal laser scanning microscopy for in vivo imaging of epidermal reactions to two experimental irritants. Skin Res Technol 14: 498-503, 2008.

60. Slodownik D, Levi A, Lapidoth M, Ingber A, Horev L and Enk CD: Noninvasive in vivo confocal laser scanning microscopy is effective in differentiating allergic from nonallergic equivocal patch test reactions. Lasers Med Sci 30: 1081-1087, 2015.

(i) (9) This work is licensed under a Creative Commons Attribution-NonCommercial-NoDerivatives 4.0 International (CC BY-NC-ND 4.0) License. 